\title{
"Drop attacks" as first clinical symptoms in a child carrying MTTK m.8344A>G mutation
}

\author{
Piotr Buda르. Dorota Piekutowska-Abramczuk², Agnieszka Karkucińska-Więckowska³, Elżbieta Jurkiewicz ${ }^{4}$, \\ Sylwia Chełstowska ${ }^{4}$, Magdalena Pajdowska ${ }^{5}$, Marek Migdał ${ }^{6}$, Janusz Książyk ${ }^{1}$, Katarzyna Kotulska ${ }^{7}$, Ewa Pronicka ${ }^{2}$ \\ ${ }^{1}$ Department of Pediatrics, Nutrition and Metabolic Diseases, ${ }^{2}$ Department of Medical Genetics, ${ }^{3}$ Department of Pathology, \\ ${ }^{4}$ Department of Radiology (MR Unit), ${ }^{5}$ Department of Biochemistry and Experimental Medicine, ${ }^{6}$ Department of Anaesthesiology \\ and Intensive Care, ${ }^{7}$ Department of Neurology and Epileptology, The Children's Memorial Health Institute, Warsaw, Poland
}

\begin{abstract}
We describe a child with dyslexia and difficulty in school who, at the age of 13 years, began to suffer from several head injuries resulting from falls of uncertain cause. Two years later, the patient developed symptoms of a severe mitochondrial disorder (involving bulbar-pyramidal paralysis, ophthalmoplegia, and hyperlactatemia) that coincided with VPA administration. Brain MR imaging revealed rapidly developing Leigh syndrome (LS), and muscle biopsy showed ragged blue fibres (RBF). A diminished expression of the Ela subunit of pyruvate dehydrogenase was found in muscle homogenate (signal $28.7 \%$ of normal).

The accurate diagnosis of mitochondrially inherited LS (MILS) and the identification of an almost homoplasmic m.8344G >A mutation in the MTTK gene was delayed due to an initial incorrect diagnosis of epilepsy, misdiagnosis of neuroinfection, and failure to note $L S$ on the first brain MRI.

Periods of exacerbation or improvement were observed in association with the administration of certain drugs or procedures (VPA administration or intensive rehabilitation associated with worsening; ketogenic diet associated with remission). However, the random association of these factors with natural disease fluctuations cannot be excluded. Conclusions: 1) To improve the early detection of mitochondrial disorder, we recommend screening for mTDNA (and $n D N A)$ mutations in all patients with LS present on brain MRI. 2) Brain MRI protocols should include diffusion-weighted and T2-weighted imaging, and LS-like changes should be analysed by a neuroradiologist experienced in the field. 3) Additional controlled studies are urgently needed to assess the causal relationship between management strategies and the natural history of the disease. Until the association between VPA and disease exacerbation can be ruled out, VPA should be avoided in patients with these symptoms unless the mitochondrial disorder has been excluded.
\end{abstract}

Key words: Leigh syndrome, MERRF mutation, pyruvate dehydrogenase dysfunction, ketogenic diet.

\section{Introduction}

Mitochondrial diseases (MID), which develop due to either mitochondrial (mtDNA) or nuclear DNA muta- tions [13], are rare. Therefore, the knowledge of these diseases among physicians may be poor, and the diagnosis of these diseases can be delayed. The extremely 
heterogeneous clinical presentation of mtDNA mutations may also exacerbate the diagnostic difficulties.

Leigh disease (LS) $[7,11]$ is a progressive subacute necrotising encephalomyelopathy characterised by focal bilateral lesions in one or more areas of the central nervous system, including the brain stem, thalamus, basal ganglia, cerebellum and spinal cord. These changes may be recognised by magnetic resonance imaging (MRI). In the majority of individuals with LS in Poland, the disease is caused by mutations in nuclear DNA (the SURF1 gene) [6] and is inherited as an autosomal recessive trait. The mitochondrially inherited form (MILS) represents only $10-20 \%$ of all LS cases $[6,7,10]$.

Although the m.8344A $>G$ mutation in the mtRNA lysine gene (MTTK) is a "common" mtDNA mutation, its prevalence was estimated as relatively low $(<1$ : 300 000) [12]. This mutation was originally described in patients with MERRF (myoclonic epilepsy with ragged red fibres) syndrome [14], but multiple heterogeneous non-MERRF presentations have been reported $[2,3,5]$. There have also been a number of descriptions of MILS related to the $m .8344 A>G$ mutation $[1,15,16]$.

The aim of this paper is to describe the first Polish patient with late-onset Leigh syndrome caused by an MTTK m.8344A>G mutation. Several factors that may have influenced the natural course of the disease, including delayed diagnosis, stress and medications used to modify the energetic metabolism of the patient, are discussed.

\section{Material and methods \\ Case report}

A 15-year-old boy on a respirator, who had been diagnosed with encephalomyelitis, was sent to $\mathrm{CMHI}$ for the inclusion in the programme of artificial ventilation at home.

The boy had developed normally during the neonatal period and infancy. His family history was negative; the parents and two older siblings are all healthy. From early childhood, the patient was under the care of a neurologist, speech therapist and psychologist due to dyslexia and school difficulties.

Beginning at 13 years of age, the patient suffered from repeated falls of uncertain cause ("drop attacks"), which were associated with several head injuries. Cardiac involvement was not observed. Valproic acid (VPA) was administered at 14 years due to suspicion of epilepsy, despite the lack of epileptiform discharges on EEG. Approximately five months after VPA administration, limb weakness, double vision, difficulties in swallowing, dyspnoea and stool incontinence appeared and rapidly developed over several weeks. Respiratory failure occurred and required artificial ventilation. Episodes of seizures, impaired consciousness, and difficulty in controlling blood pressure were observed at a local paediatric ward. Plasma lactate levels were slightly elevated $(24 \mathrm{mg} / \mathrm{dl}$, ref. $<20 \mathrm{mg} / \mathrm{dl})$. Liver enlargement $(6 \mathrm{~cm})$, mild transaminase elevation ( $<100 \mathrm{U} / \mathrm{l}$ ) and hyperammonaemia $(300 \mu \mathrm{g} / \mathrm{dl})$ were found. Initially, neuroinfection was suspected based on the results of borreliosis testing (IgG + antibodies), and relevant therapy was applied. Repeated attempts at extubation failed.

At the age of 15 years, the patient was admitted to the $\mathrm{CMHI}$ intensive care ward; his physical development was normal (height $180 \mathrm{~cm}$, weight $63 \mathrm{~kg}$ ). Due to the initial suspicion of undetermined neuroinfection, the patient was treated with intravenous immunoglobulins, antibiotics, and steroids. VPA administration was discontinued, and carnitine, vitamin $B_{1}$ and coenzyme $Q$ were administered. His clinical condition improved quickly, and after ten days, the boy was weaned from mechanical ventilation and transferred to the neurological ward. Neurological examination revealed the severe bulbo-pyramidal syndrome. Visual evoked potentials were abnormal.

Laboratory examination did not reveal any signs of infection. The lactate concentration in the plasma $(58.3 \mathrm{mg} / \mathrm{dl})$ was high, and that in the cerebrospinal fluid was in the upper range of normal $(22.6 \mathrm{mg} / \mathrm{dl})$. The organic acid profile revealed an increased excretion of lactate and Krebs cycle metabolites as well as the presence of VPA derivatives in the urine.

This time, a mitochondrial disorder was suspected, and an open muscle biopsy was performed, confirming mitochondrial pathology. The clinical condition of the patient showed further gradual improvement (he became able to walk with support), and he was discharged home.

A few months later, the boy was re-admitted to $\mathrm{CMHI}$ due to further deterioration in speech and movement as well as dyspnoea. Bulbar and pseudobulbar palsy, tetraparesis, generalised hypotonia, absent deep tendon reflexes, and pathologic reflexes were exacerbated during the implementation of an intensive rehabilitation program. The rehabilitation was then stopped, and his neurolog- 
ical status slowly improved. The boy was conscious and well oriented at all times.

After a short period of relative remission at home, rapid neurological progression recurred at the age of 15.5 years. Mechanical ventilation was required for one week. A significant worsening of the patient's condition during infusion of mannitol and glucose was observed. Because these symptoms were noted at least twice, an intravenous glucose tolerance test (IVGTT) was conducted. An abnormal increase in the lactate level (in response to hyperglycaemia) was detected during the test (17 and $36 \mathrm{mg} / \mathrm{dl}$ before and 30 min after load, respectively), indicating a dysfunction at the level of the PDH (pyruvate dehydrogenase) complex.

When the diagnosis of the mitochondrial disorder with poor prognosis was established, the patient and his parents chose not to continue insistent therapy. The boy was discharged home under the home care service of local hospice. There, a ketogenic diet was introduced through the gastrostomy (PEG) tube, and supplementation with coenzyme $\mathrm{Q}$, carnitine and vitamin $B_{1}$ (reported to have some beneficial effect in PDH and mitochondrial dysfunction) was continued. The neurological status of the patient stabilised for several months. The boy did not require mechanical ventilation and was able to talk. He experienced some difficulties in swallowing, and therefore a PEG tube was often used for feeding. He required a wheelchair. The patient gained weight properly and his laboratory tests did not show significant abnormalities. Plasma lactate levels remained normal or at the upper normal limit.

One year later, at the age of 16.5 , the boy experienced a stroke-like episode. He died at home at the age of 17 years.

This investigation conformed to the principles outlined in the Declaration of Helsinki, and the study protocol was approved by the Bioethical Commission of the Children's Memorial Health Institute.

\section{Brain magnetic resonance imaging and spectroscopy}

MR brain and spinal cord examinations were acquired with $1.5 \mathrm{~T}$ scanners. The brain protocol involved the acquisition of T1- and T2-weighted images, FLAIR sequence, and diffusion-weighted images (DWI). Two spinal cord images (T1- and T2-weighted) were obtained in two planes each. Contrast-enhanced T1-weighted images were acquired in all studies.
In vivo multi-voxel proton magnetic resonance spectroscopy $\left({ }^{1} \mathrm{H}-\mathrm{MRS}\right)$ was also performed. We used a PRESS 2D-chemical shift imaging (CSI) sequence with the following parameters: $T R=1500 ; T E=30$ with planar resolution; FOV, $230 \mathrm{~mm}$; matrix size, $16 \times 16 \mathrm{~cm}^{2}$; section thickness, $2 \mathrm{~cm}$. Full analyses of the spectra were performed in LC Model software.

\section{Muscle biopsy}

A mitochondrial study of the muscle biopsy was performed according to histochemical and spectrophotometric methods commonly used in our laboratory. The assessment included light microscopic examination of frozen sections in the following stains and histochemical reactions: haematoxylin and eosin (HE), modified Gomori trichrome, oil red O, succinate dehydrogenase, NADH dehydrogenase, cytochrome $c$ oxidase (COX), acid phosphatase, myosin ATP-ase preincubated at $\mathrm{pH}$ 4.3, 4.6 and 9.4. The activity of respiratory chain complexes I-IV was measured in the muscle homogenate as described [6].

\section{DNA analysis}

Total DNA was extracted from the whole blood of the patient, his two siblings and his mother using a standard phenol/chloroform extraction procedure. The SURF1, MTATP6 and MTTK genes were amplified and sequenced in 3130 Genetic Analyzer with BigDye Terminators (Applied Biosystems). Multiplex Ligation Probe Amplification (MLPA) analysis was performed with the SALSA MLPA P125-B1 Mitochondria kit (MRC-Holland) according to the manufacturer's protocol.

\section{Samples preparation for Western blot}

Tissue samples kept at $-80^{\circ} \mathrm{C}$, were defrost, cut into small pieces and resuspended in cold lysis buffer (50 mM Tris pH 7.5, $150 \mathrm{mM} \mathrm{NaCl}, 1 \%$ Triton, $0.1 \%$ SDS, $1 \%$ sodium deoxycholate) containing inhibitors of proteases (1 mM PMSF and the protein protease inhibitor cocktail). Skeletal muscles were homogenized on ice. Samples were incubated on ice for $20 \mathrm{~min}$ and centrifuged at $600 \times \mathrm{g}$ for $5 \mathrm{~min}$ at $4^{\circ} \mathrm{C}$ to remove insoluble material. The supernatants were collected and centrifuged at $16000 \times \mathrm{g}$ for $20 \mathrm{~min}$ at $4^{\circ} \mathrm{C}$. Protein concentration in lysates was determined using Bradford method. Samples for SDS-PAGE were denatured in reducing Laemmli loading buffer at $95^{\circ} \mathrm{C}$ for $5 \mathrm{~min}$. 


\section{Western blotting}

Tissue lysates (20 $\mu \mathrm{g}$ protein) from muscles (patient and healthy control) were separated electrophoretically in $8 \%$ SDS polyacrylamide gels and transferred into PVDF membrane (BioRad). Membranes were blocked and proteins were detected using MitoProfile ${ }^{\circledR}$ Pyruvate dehydrogenase (PDH) WB Antibody Cocktail (abcam) followed by appropriate secondary AP-conjugated antibodies (1 : 1000, Bio-Rad).

\section{Results \\ Electrophysiological findings}

The available EEG recordings from the patient demonstrate progressive pathology: periodic epileptiform discharges (PEDs) at the age of 15 years, triphasic periodical waves and no spikes after few weeks, and the presence of frontal intermittent rhythmic delta activity (FIRDA) two months later.

Evoked potentials examined at the first rapid deterioration, at the age of 15 years, revealed abnormal visual evoked potentials. Auditory potentials were not recorded.

\section{Magnetic resonance imaging and spectroscopy}

MR examination was performed three times over a year to document the clinical progression of the disease. Re-assessment of these MR images showed that lesions suggesting LS were already present at the first examination, at the age of 14.5 years (not shown). They were milder than those found 6 months later.

The MR examinations at the age of 15 years are shown in the upper portion of Fig. 1 (panels A-D). The co-occurrence of bilateral, symmetrically located lesions in the thalamic region were not mentioned in the first description of the results, although they are clearly visible on diffusion-weighted images (Fig. 1B). Spinal cord imaging performed 10 days later (Fig. 1C-D) showed a diffuse abnormal signal in the medulla oblongata, cervical spinal cord and upper portion of the thoracic spinal cord (Th1-Th3).

Follow-up brain and spinal canal MR exams taken at the age of 15.5 years revealed a remarkable progression of both localisations. The bilateral thalamus, putamen, midbrain, pons, medulla oblongata, and entire length of the spinal cord were involved. The lesions were observed predominantly on T2- and diffusion-weighted images in the brain (Fig. 1E-F) and on T2-weighted images in the spinal cord (Fig. $1 \mathrm{G}-\mathrm{H})$.

MR spectroscopy with the voxel localised in the right putamen demonstrated significantly elevated lactate levels and relatively low levels of Cho (Cho/ $\mathrm{Cr}=0.26)$ and NAA (NAA/Cr = 0.94). The levels of Glx $(\mathrm{Glx} / \mathrm{Cr}=2.7)$ and $\mathrm{ml}(\mathrm{ml} / \mathrm{Cr}=0.81)$ were relatively elevated (Fig. 2).

\section{Histochemical and biochemical findings}

Histochemistry of muscle samples revealed few distinct ragged blue fibres (RBF) (Fig. 3) accompanied by a pattern suggestive of mosaic COX deficiency and mild to moderate lipid accumulation. No classical ragged red fibres (RRF) were observed.

Spectrophotometry of a muscle homogenate showed an increased citric synthase activity $(267.5 \mathrm{nmol} /$ $\mathrm{min} / \mathrm{mg}$ protein, ref. < $180 \mathrm{nmol} / \mathrm{min} / \mathrm{mg}$ protein) and normal activity for respiratory chain complexes I-IV (not shown).

Immunoblotting with pyruvate dehydrogenase (PDH) complex antibodies showed a diminished signal representing the steady state level of the E1 $\alpha$ subunit in the muscle homogenate $(28.7 \%$ of the reference value), consistent with PDH dysfunction (Fig. 4).

\section{DNA analysis}

A search for the common mutations responsible for LS in the SURF1 and MTATP6 genes was negative. MLPA analysis demonstrated a lack of ligation of the m.8344A $>\mathrm{G}$-specific probe. Further, MTTK gene sequencing confirmed the presence of the m.8344A $>G$ mutation in $96 \%$ of his mtDNA. The heteroplasmy levels of this mutation in the patient's sister and mother were $80 \%$ and $70 \%$, respectively (all family members were asymptomatic).

\section{Discussion}

The patient bearing an m.8344G $>A$ mutation (proband) rapidly developed a full complement of symptoms of the mitochondrial disorder, including muscle weakness, loss of acquired skills, bulbar-pyramidal syndrome, ophthalmoplegia, Leigh syndrome, stroke-like episodes, elevated lactate concentration, and ragged blue muscle fibres. The first exacerbation (with transient liver enlargement and dysfunction) coincided with VPA administration. The course of the 


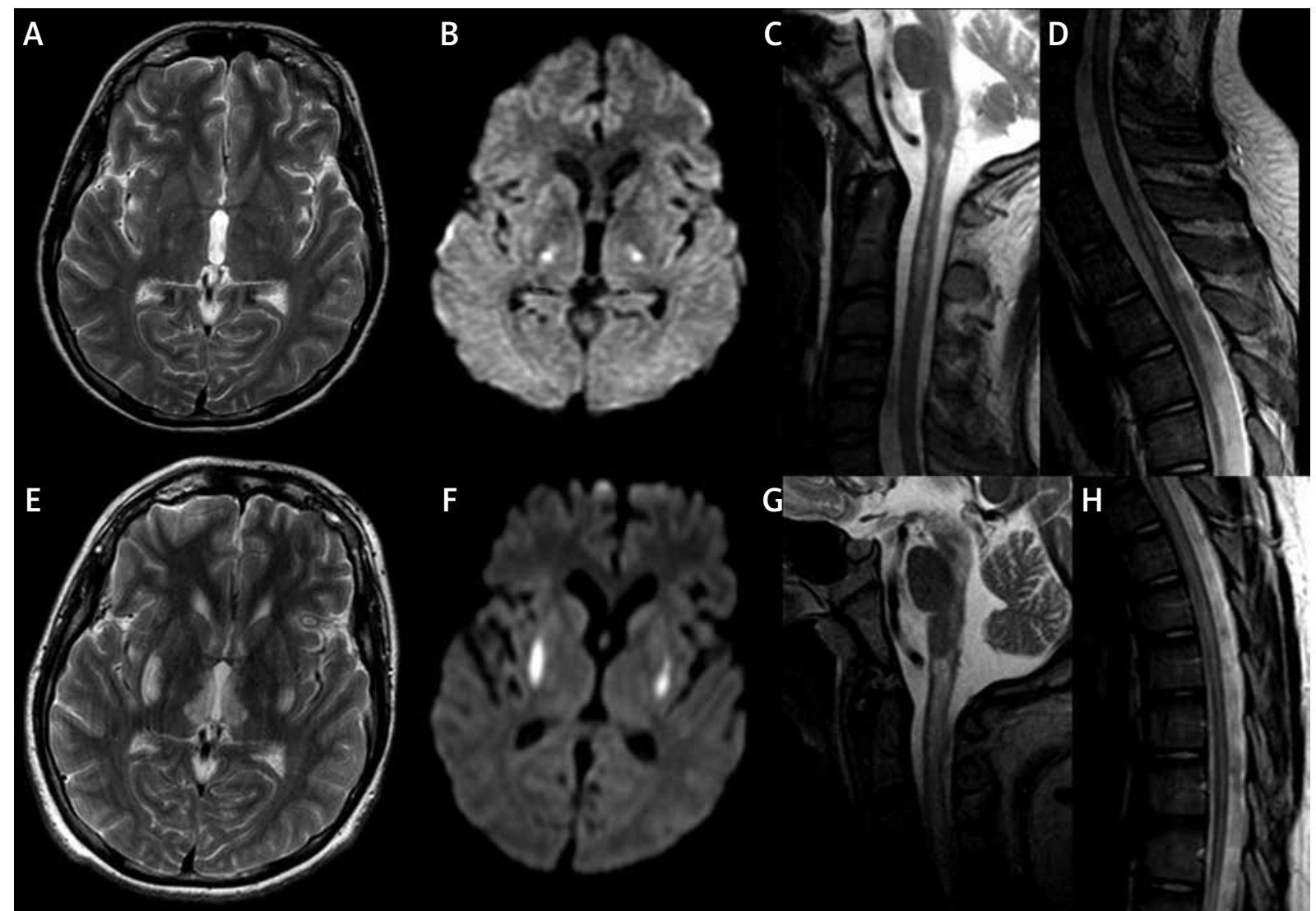

Fig. 1. MR examination performed at the age of 15 years. Axial planes. A) T2-weighted image, B) diffusion-weighted image. Abnormal, symmetric hyperintensities in the thalami, better seen on B sagittal planes. C-D) T2-weighted images. Hyperintense signal in the medulla oblongata and cervical spinal cord up to the upper level of C3 is seen. The second lesion involved the spinal cord from Th1 to Th3. MR study performed 6 months later revealed a significant progression. Axial planes. E) T2-weighted image, F) diffusion-weighted image. Bilateral lesions were located in both putamen and thalami with restricted diffusion in the putamen (seen on DWI). Sagittal planes. G-H) T2-weighted images. Abnormal signal involved midbrain, posterior part of pons, medulla oblongata, cervical and thoracic spinal cord.

disease had overlapping symptoms of MILS, MERRF and MELAS (mitochondrial encephalopathy, lactic acidosis, stroke-like episodes).

Leigh syndrome (LS) is a frequent presentation of the mitochondrial disorder in children, and it occurs regardless of the molecular aetiology of the mitochondrial pathology. The disease has a peculiar topography and specific pathological morphology [7]. It is not yet understood why LS develops rarely in mitochondrial tRNA-mutated patients [16], more frequently in PDH-deficient patients, and most frequently in SURF1-mutated patients [6].

According to our hypothesis, episodes of hyperventilation and hypocapnia increase the risk of LS development [9]. A transient elevation in the intracellular $\mathrm{pH}$ of neurons (respiratory alkalosis) could trigger apoptosis in specially structured regions of the brain affected in LS.

We documented the occurrence of respiratory alkalosis in SURF1-related LS and also described this phenomenon in an LS patient with another MTTK m.8363 mutation [10]. Alkalosis and hypocapnia were not documented in the reported patient, but the occurrence of hyperventilation episodes due to anxiety over his unexpectedly rapid deterioration cannot be excluded.

The proper diagnosis of our patient was delayed by an incorrect recognition of epilepsy and the administration of VPA (a known mitochondrial suppressor), misdiagnosis of infectious encephalomyelitis, and the failure to note the LS in the first brain MRI performed at the local paediatric ward. It should be 


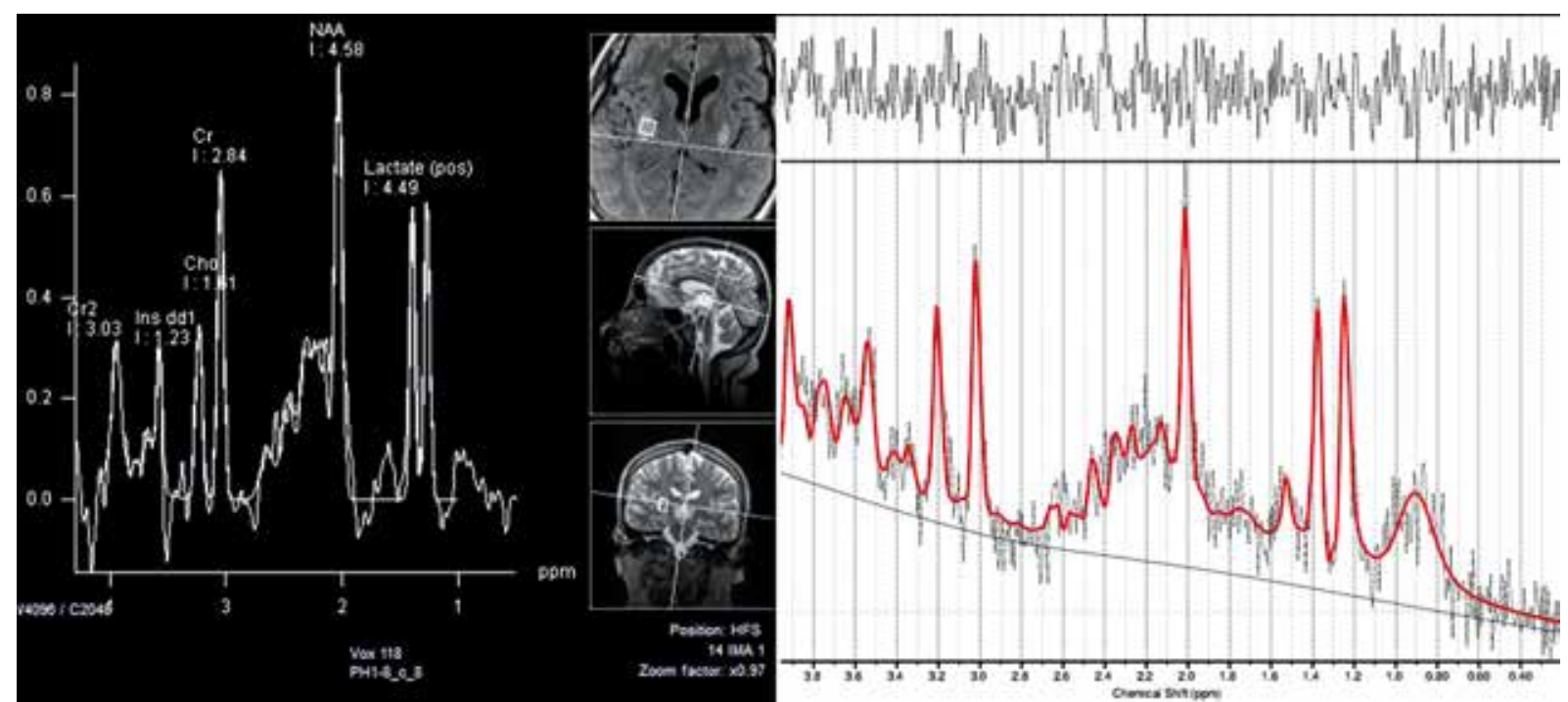

Fig. 2. Multi-voxel proton spectroscopy, PRESS acquisition (TR/TE 1500/30) at the level of right putamen at the age of 15.5 years demonstrates elevated lactate levels.

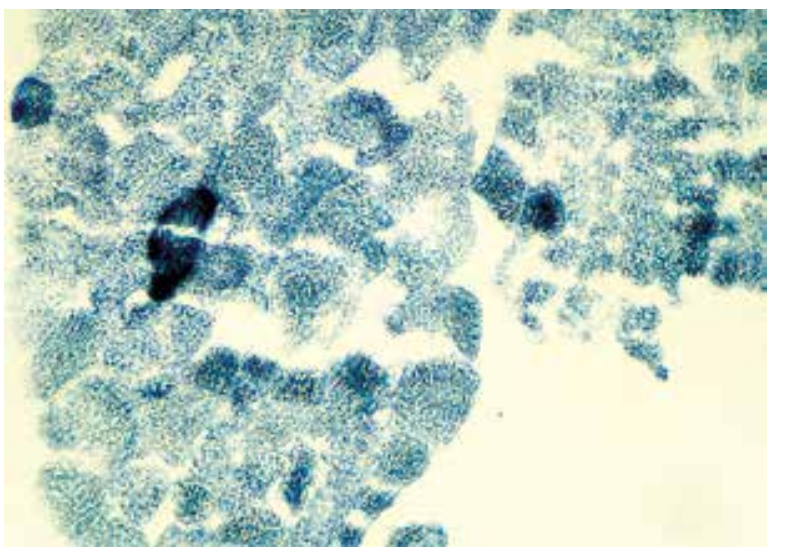

Fig. 3. Skeletal muscle biopsy. Succinate dehydrogenase reaction showing few ragged blue fibers. Original magnification 200x.

emphasised that the m.8344A $>G$ mutation could be detected much earlier because the variant is included in DNA screening panel recommended for LS [6]. Early diagnosis could protect the patient from unnecessary procedures and harmful medications. Further, early diagnosis might have prevented (slowed down or even stopped) the rapid progression of LS changes demonstrated in subsequent brain MR examinations.

Interestingly, some features of PDH deficit, including glucose intolerance and low Elalfa subunit expression, which is also known to cause LS [7,11,17], were observed in our patient. Similarly impaired PDH activity was previously described in a patient with an MTTL m.3243A>G mutation [18]. Both of these mtDNA mutations disrupt mitochondrial tRNAs (for lysine and leucine, respectively) and impair mitochondrial protein synthesis $[1,18,19]$. A disruption of the import and/or the misassembly of cytosolic synthesised polypeptides was suggested to explain the PDH defect in the m.3243A>G mutated cells [18].

The undulating course of the disease, which is a major hallmark of mitochondriopathies, was evident in the reported patient. This severely complicated the evaluation of the therapeutic approaches used. The periods of exacerbation or improvement in our patient were apparently associated with the drugs or medical procedures applied. It is tempting to suggest a causal relationship, e.g. between the administration of VPA [8] (or intensive rehabilitation) and the clinical deterioration or between the diet manipulation [4,17] and the remission; however, neither could be conclusively proven in this patient. It should be remembered that random independent fluctuations in the clinical course may imitate a good or bad response to parallel treatments.

Our expanding knowledge of the natural history of m.8344A $>$ G-related disease reveals a very broad range of symptoms and severity $[2,3,5]$, regardless of the heteroplasmy level. Multiple environmental factors (favourable or damaging) may influence the course of disease in individual patients [4]. This allows speculation that avoiding harmful factors can slow down disease progression, and vice versa. Until 
A

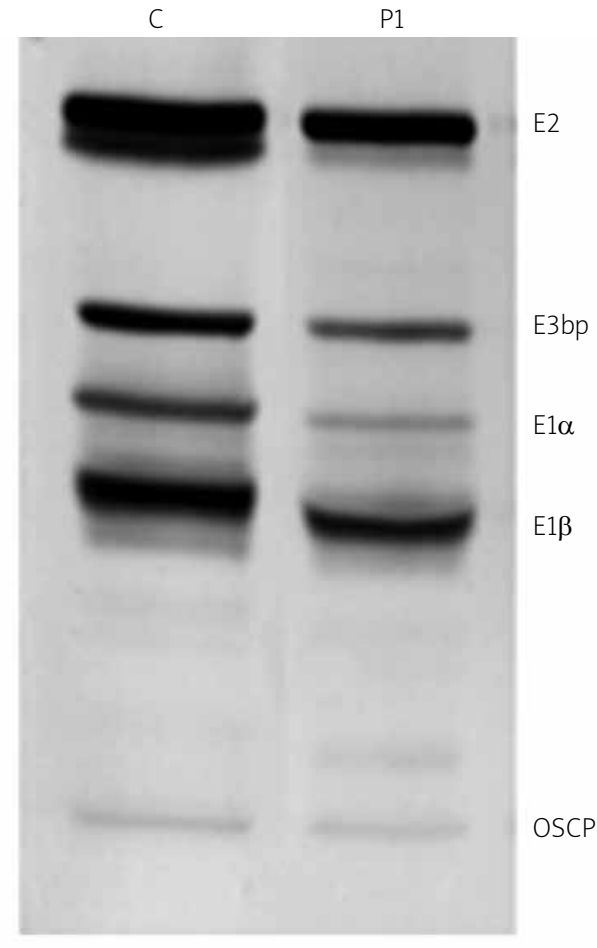

the nature of these relationships is finally established, the potential impacts of treatment must be considered in the management of patients with suspected mitochondrial disease [4].

In summary: We recommend molecular screening for mtDNA (and nDNA) mutations in all patients with symmetric lesions in the subcortical nuclei and other locations characteristic of LS present on brain MR examination.

The brain MRI protocol should obligatorily include diffusion-weighted (DWI), and T2-weighted (T2-WI) imaging; LS-like changes should be assessed (or at least consulted on) by a neuroradiologist experienced in the field.

Valproic acid (VPA) should be avoided as an anticonvulsant drug in patients with a suspected mitochondrial disorder. In cases of severe epilepsy, especially associated with other symptoms of encephalopathy, a mitochondrial pathology of any kind should be carefully considered (i.e. according to Nijmegen criteria) before VPA introduction.

The benefit of a ketogenic diet for mitochondrial tRNA-mutated patients cannot be assessed until prospective blinded studies in larger groups of patients are performed.
B

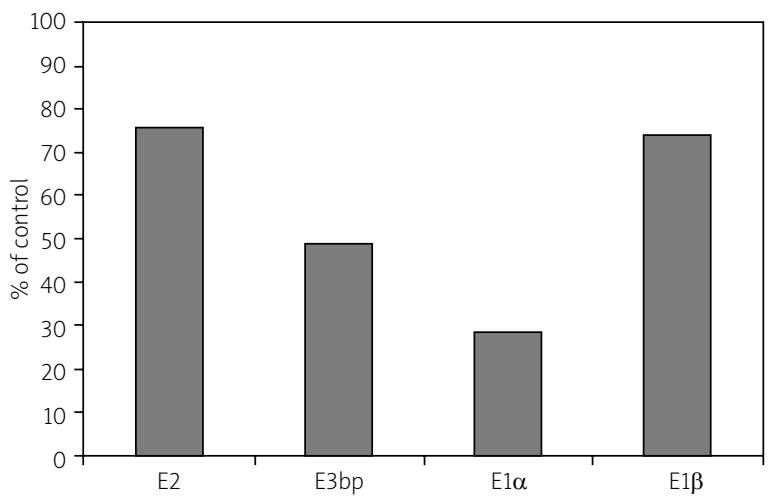

Fig. 4. Level of selected subunits of pyruvate dehydrogenase complex (E1 $\alpha, E 1 \beta, E 3 b p$ and E2) from skeletal muscle obtained in biopsy of patients (P1) and control samples of muscle (K). A) Representative western blot of selected subunits of pyruvate dehydrogenase. B) Densitometric analysis of selected subunits of the pyruvate dehydrogenase level for the patient. The levels of individual subunits were calculated as a ratio to corresponding subunits in control tissue.

\section{Acknowledgements}

The work was supported by the grant of the Children's Memorial Health Institute for young researchers no. M2/2012.

\section{References}

1. Chinnery PF, Howell N, Lightowelers RN, Turnbull DM. Molecular pathology of MELAS and MERRF. The relationship between mutation load and clinical phenotypes. Brain 1997; 120: 1713-1721.

2. Erol I, Alehan F, Horvath R, Schneiderat P, Talim B. Demyelinating disease of central and peripheral nervous systems associated with a A8344G mutation in tRNALys. Neuromuscul Disord 2009; 19: 275-278.

3. Horvath R, Kley RA, Lochmüller H, Vorgerd M. Parkinson syndrome, neuropathy, and myopathy caused by the mutation A8344G (MERRF) in tRNALys. Neurology 2007; 68: 56-58.

4. Koene S, Smeitink J. Metabolic manipulators: a well founded strategy to combat mitochondrial dysfunction. J Inherit Metab Dis 2011; 34: 315-325.

5. Molnar MJ, Perenyi J, Siska E, Nemeth G, Nagy Z. The typical MERRF (A8344G) mutation of the mitochondrial DNA associated with depressive mood disorders. I Neurol 2009; 256: 264-265.

6. Piekutowska-Abramczuk D, Popowska E, Pronicki M, Karczmarewicz E, Tylek-Lemanska D, Sykut-Cegielska J, SzymanskaDembinska T, Bielecka L, Krajewska-Walasek M, Pronicka E. 
High prevalence of SURF1 C.845_846delCT mutation in Polish Leigh patients. Eur J Pediatr Neurol 2009; 13: 145-153.

7. Piekutowska-Abramczuk D. The molecular background of Leigh syndrome. Neurol Neurochir Pol 2008; 42: 238-250.

8. Pronicka E, Weglewska-Jurkiewicz A, Pronicki M, Sykut-Cegielska J, Kowalski P, Pajdowska M, Jankowska I, Kotulska K, Kalicinski P, Jakobkiewicz-Banecka J, Wegrzyn G. Drug-resistant epilepsy and fulminant valproate liver toxicity. Alpers-Huttenlocher syndrome in two children confirmed post mortem by identification of p.W748S mutation in POLG gene. Med Sci Monit 2011; 17: 203-209.

9. Pronicka E, Piekutowska-Abramczuk D, Popowska E, Pronicki M, Karczmarewicz E, Sykut-Cegielska J, Taybert J. Compulsory hyperventilation and hypocapnia of patients with Leigh syndrome associated with SURF-1 gene mutations as a cause of low serum bicarbonates. I Inherit Metab Dis 2001; 24: 707-714.

10. Pronicki M, Sykut-Cegielska J, Matyja E, Musialowicz J, Karczmarewicz E, Tonska K, Piechota J, Piekutowska-Abramczuk D, Kowalski P, Bartnik E. G8363A mitochondrial DNA mutation is not a rare cause of Leigh syndrome - clinical, biochemical and pathological study of an affected child. Folia Neuropathol 2007; 45: 187-191.

11. Rahman S, Blok RB, Dahl HH, Danks DM, Kirby DM, Chow CW, Christodoulou J, Thorburn DR. Leigh syndrome: clinical features and biochemical and DNA abnormalities. Ann Neurol 1996; 39: 343-351.

12. Remes AM, Karpa M, Rusanen H, Majamaa K. Epidemiology of the mitochondrial DNA 8344A>G mutation for the myoclonus epilepsy and ragged red fibers (MERRF) syndrome. J Neurol Neurosurg Psychiatry 2003; 74: 1157-1161.

13. Shapira AHV. Mitochondrial disease. Lancet 2006; 368: 70-82.

14. Silvestri G, Ciafoloni E, Santorelli FM, Shanske S, Servidei S, Graf WD, Sumi M, DiMauro. Clinical features associated with the $A \rightarrow G$ transition at nucleotide 8344 of mtDNA ("MERRF mutation"). Neurology 1993; 43: 1200-1206.

15. Swiderska N, Appleton R, Morris A, Isherwood D, Selby A. A novel presentation of inappropriate antidiuretic hormone secretion in Leigh syndrome with the myoclonic epilepsy and ragged red fibers, mitochondrial DNA 8344A>G mutation. J Child Neurol 2010; 25: 782-785.

16. Tsao CY, Herman G, Boué DR, Prior TW, Lo WD, Atkin JF, Rusin J. Leigh disease with mitochondrial DNA A8344G mutation: case report and brief review. J Child Neurol 2003; 18: 62-64.

17. Wexler ID, Hemalatha SG, McConnell J, Buist NR, Dahl HH, Berry SA, Cederbaum SD, Patel MS, Kerr DS. Outcome of pyruvate dehydrogenase deficiency treated with ketogenic diets. Studies in patients with identical mutations. Neurology 1997; 49: 1655-1661.

18. Wilichowski E, Korenke GC, Ruitenbeek W, De Meirleir L, Hagendorff A, Janssen AJ, Lissens W, Hanefeld F. Pyruvate dehydrogenase complex deficiency and altered respiratory chain function in a patient with Kearns-Sayre/MELAS overlap syndrome and A3243G mtDNA mutation. J Neurol Sci 1998; 157: 206-213.

19. Wu SB, Ma YS, Wu YT, Chen YC, Wei YH. Mitochondrial DNA mutation - elicited oxidative stress, oxidative damage, and altered gene expression in cultured cells of patients with MERRF syndrome. Mol Neurobiol 2010; 41: 256-266. 